\title{
ON STOPPING TIME DIRECTED CONVERGENCE
}

\author{
BY ARYEH DVORETZKY \\ Communicated by Alexandra Bellow, December 31, 1975
}

The main purpose of this note is to introduce the notion of $\bar{S}$-martingales, a certain modification of that of asymptotic martingales, the main justification of which is III.

1. $S$-convergence. Let $(\Omega, F, P)$ be a probability; $\left(F_{n}\right)(n=1,2, \ldots)$, a nondecreasing sequence of measurable $\sigma$-fields and $\left(X_{n}\right)$ an adapted sequence of extended real-valued r.v. (random variables). (If the $F_{n}$ are not mentioned explicitly then any $F_{n}$ with the above properties will do; in particular, we may take $F_{n}$ to be the $\sigma$-field generated by $X_{1}, \ldots, X_{n}$.) Let $T=\{t\}$ be the family of bounded stopping times; i.e. the family of positive, bounded, integer-valued r.v. $t$ with $t^{-1}(n) \in F_{n}$ for all $n$. $T$ is a directed set filtering to the right under the relation $t_{1} \leqslant t_{2}$, i.e. $t_{1}(\omega) \leqslant t_{2}(\omega)$ a.s. (almost surely). The r.v. $X_{t}$ for $t \in T$, is defined by $X_{t}(\omega)=X_{t(\omega)}(\omega)$.

Definition. Let $\phi$ map $X_{t}(t \in T)$ into a topological space $M$. Then $\left(\phi\left(S_{n}\right)\right)$ is said to be $S$-convergent-or stopping time directed convergent-(to $Y$ ) if the directed set $\phi\left(X_{t}\right)$ is convergent in the topology of $M$ (to $Y$ ).

$S$-convergence implies ordinary convergence, but not vice-versa.

EXAMPLES. (1) $\phi$ the identity mapping, $M$ the space of all extended real valued r.v. topologized by convergence in probability (for extended real valued r.v. this is interpreted as applied to the r.v. obtained through the mapping $x \rightarrow$ $x /(1+|x|))$. We then speak of $S$-convergence in probability. In sharp distinction from the situation in ordinary convergence, we have

I. S-convergence in probability is equivalent to a.s. convergence.

The proof is immediate since there exist $t_{1}<\cdots<t_{n}<\cdots \rightarrow \infty$ with $X_{t_{n}} \stackrel{\text { a.s }}{\rightarrow} \lim \sup X_{t_{n}}=\lim \sup X_{n}$.

(2) $\left(X_{n}\right)$ is said to be an $S$-martingale if the expectations (finite or not) $E X_{t}$ are defined for all $t \in T$ and $\left(E X_{n}\right)$ is $S$-convergent (to a finite or infinite number). If the limit is a finite number then $\left(X_{n}\right)$ is called an asymptotic martingale.

The argument proving I yields

II. A uniformly bounded sequence of r.v. $\left(X_{n}\right)$ is a.s. convergent iff it is an asymptotic martingale.

AMS (MOS) subject classifications (1970). Primary 60G40, 60G45; Secondary 28A65, 28A20. 
This result is due to P. A. Meyer [5] where, apparently, stopping time directed convergence was first considered in this context. Asymptotic martingales were introduced by D. G. Austin, G. A. Edgar and A. Ionescu Tulcea [1] and their properties further studied and importance underlined by R. V. Chacon and L. Sucheston [3], G. A. Edgar and L. Sucheston [4] and A. Bellow [2].

2. $\bar{S}$-convergence. Let $C^{H}(0 \leqslant H<\infty)$ be defined by: $C^{H}(x)=x$ if $|x| \leqslant H$ and $C^{H}(x)= \pm H$ otherwise, according to the sign of $x$.

DEFINITION. Let $\varphi$ map uniformly bounded r.v. into the topological space $M$. Then $\left(\phi\left(X_{n}\right)\right)$ is said to be $\bar{S}$-convergent if $\phi\left(C^{H} X_{n}\right)$ is $S$-convergent for every $H$.

In particular, $\left(X_{n}\right)$ is said to be an $\bar{S}$-martingale if $\left(C^{H} X_{n}\right)$ is an asymptotic martingale for every $H . \bar{S}$-convergence in probability is defined as $S$-convergence in probability of $\left(C^{H} X_{n}\right)$ for every fimite positive $H$.

From II we immediately have

III. A sequence of extended real-valued r.v. $\left(X_{n}\right)$ is a.s. convergent iff it is an $\bar{S}$-martingale.

It follows (and can also be shown directly) that in defining $\bar{S}$-martingales it suffices to consider any unbounded set of $H$ 's. On the other hand, one can equivalently define $\bar{S}$-martingales by the requirement that $\left(f\left(X_{n}\right)\right)$ is an asymptotic martingale for every bounded continuous function $f$ from the extended real line into the real line.

We remark that

IV. The limit function in III is a.s. finite iff

$$
\lim _{H \rightarrow \infty} \limsup _{n \rightarrow \infty} P\left[\left|X_{n}\right|>H\right]=0
$$

From III we have immediately

V. (a) If $\left(X_{n}\right)$ and $\left(Y_{n}\right)$ are $\bar{S}$-martingales, then $\left(\min \left(X_{n}, Y_{n}\right)\right)$ and $\left(\max \left(X_{n}, Y_{n}\right)\right)$ are also $\bar{S}$-martingales.

(b) A function $f$ transforms every $\bar{S}$-martingale $\left(X_{n}\right)$ into an $\bar{S}$-martingale $\left(f\left(X_{n}\right)\right)$ iff it is continuous (on the extended real line).

(c) If $P^{\prime}$ is a probability measure defined on $F$ then every $\bar{S}$-martingale on $(\Omega, F, P)$ is an $\bar{S}$-martingale on $\left(\Omega, F, P^{\prime}\right)$ iff $P^{\prime}$ is absolutely continuous relative to $P$.

3. Further results. In [1] (see also [4], [2]) it was proved that an $L_{1}$ bounded asymptotic martingale is a.s. convergent. This implies

VI. An $L_{1}$-bounded asymptotic martingale is an $\bar{S}$-martingale.

This also follows directly from VII, which extends a result of [1].

VII. If $\left(X_{n}\right)$ is an asymptotic martinglae then $\left(X_{n}^{+}\right)$and $\left(X_{n}^{-}\right)$are S-martingales.

To see this note that if $t^{\prime} \geqslant t$ and we put $t^{\prime \prime}=t^{\prime}$ on $\left[X_{t} \geqslant 0\right]$ and $t^{\prime \prime}=t$ otherwise, then $t^{\prime \prime} \geqslant t$. Hence, if $t$ is such that $E X_{t^{\prime}}-E X_{t} \geqslant-\epsilon$ for all $t^{\prime} \geqslant$ 
$t$, it follows that $E X_{t^{\prime}}^{+}-E X_{t}^{+} \geqslant E X_{t^{\prime \prime}}^{+}-E X_{t}^{+} \geqslant E X_{t^{\prime \prime}}-E X_{t} \geqslant-\epsilon$.

We remark that the conclusion of VII holds even for $S$-martingales which are not asymptotic martingales, provided the approach of $E X_{t}$ to infinity is "semimonotone". In particular it holds for super- and sub-martingales (even not $L_{1}$-bounded).

4. Generalizations. There are several possible ways of generalizing the above results. Thus (1) more general directed sets than sequences can be considered (see [4]). (2) Vector valued (and other) r.v. may be considered (see [3], [4], [2] ).

(3) Probability spaces can be replaced by $\sigma$-finite measure spaces. (4) Approximate (see [4]) $S$ - and $\bar{S}$-martingales can be studied.

ACKNOWLEDGements. My interest in the subject was stimulated by the Colloquium lecture of Alexandra Bellow in Jerusalem mentioned in [2]. I am also indebted to here for illuminating discussions as well as for prompting me to write this note. I would like also to thank G. A. Edgar and L. Sucheston for making [4] available before publication.

\section{REFERENCES}

1. D. G. Austin, G. A. Edgar and A. Ionesco Tulcea, Pointwise convergence in terms of expectations, Z. Wahrscheinlichkeitstheorie und Verw. Gebiete 30 (1974), 17-26.

2. A. Bellow, Stability properties of the class of asymptotic martingales, Bull. Amer. Math. Soc. 82 (1976), 338-340.

3. R. V. Chacon and L. Sucheston, On convergence of vector-valued asymptotic martingales, Z. Wahrscheinlichkeitstheorie und Verw. Gebiete 33 (1975), 55-59.

4. G. A. Edgar and L. Sucheston, Amarts, a class of asymptotic martingales (Discrete parameters), J. Multivariate Anal. (to appear).

5. P. A. Meyer, Probability and potentials, Blaisdell, Waltham, Mass., 1966. MR 34 \#5119.

HEBREW UNIVERSITY OF JERUSALEM, JERUSALEM, ISRAEL 